\title{
An Energy Balancing Approach for MANETs Based on Non-cooperative Game
}

\author{
Xin $\mathrm{LI}^{1}$ Huyin $\mathrm{ZHANG}^{1}$ Di WU ${ }^{1}$ Jing WANG ${ }^{1}$ \\ ${ }^{1}$ School of Computer \\ Wuhan University \\ Wuhan City, Hubei Province, China \\ lixin@whu.edu.cn
}

\begin{abstract}
In a mobile Ad Hoc network, the energy imbalance of mobile nodes may lead to energy depletion of some nodes, network partitioning and degradation of network lifespan. Therefore, we propose an energy balancing approach called Energy Balancing Approach Based on Non-cooperative Game (EBNG) which can be transparently added to any existing reactive routing protocols. EBNG combines an Energy Balancing Approach Based on Forwarding Refusal (EBFR) which allows nodes to give up packet forwarding depending on their battery power with a Protocol-Independent Cooperative Incentive Scheme (PICIS) which under a dynamic repeated game-theoretic framework. Simulation results show that compared with the existing schemes EBFR can extend the lifespan of MANET and PICIS performs well in terms of the effectiveness of cooperation incentives and selfish node detection.
\end{abstract}

Keywords-Mobile Ad Hoc Network; Energy Balancing; Selfish Node; Non-cooperative Game

\section{INTRODUCTION}

Mobile ad hoc networks (MANETs) are multi-hop mobile wireless networks which do not have any preexisting network infrastructure or centralized administration ${ }^{[1]}$. In MANETs, routes can be used for long times until they become stale. This will result in the nodes on the shortest path consume more power to forward others' packets then deplete their energy earlier than other nodes. As a result, the lifespan of MANETs becomes far shorter and entire network will be split into several subnets. Although this problem is very serious, most solutions do not take link capacity and energy consumption into consideration ${ }^{[2]}$.

In this paper, we propose an energy balancing approach called Energy Balancing Approach Based on Noncooperative Game (EBNG) which combines an Energy Balancing Approach Based on Forwarding Refusal (EBFR) with a Protocol-Independent Cooperative Incentive Scheme (PICIS). EBFR tries to extend lifespan of MANETs by reducing packet forwarding of low-energy nodes to prevent traffic concentration. We propose PICIS which under a dynamic repeated game-theoretic framework to encourage nodes to participate in forwarding packets voluntarily. Owing to use punishment for non-cooperation, all nodes in MANET are willing to cooperate with each other. It is shown from the simulation results that compared with the existing schemes EBFR can extend the lifespan of MANET and PICIS performs well in terms of the effectiveness of cooperation incentives and selfish node detection.
The remainder of the paper is organized as follows: Section 2 provides an introduction to the related work. In Sections 3 and 4, we describe the operations of EBFR and PICIS respectively. In addition, the simulation results are presented and analysed in Section 5. Then we conclude the paper in Section 6.

\section{RELATED WORK}

Younghwan Yoo et al. ${ }^{[3]}$ propose a load balancing approach called Simple Load Balancing Approach (SLBA), which can be transparently added to any current reactive routing protocol. SLBA can distribute traffic very well and improve the MANET performance. In [4] the authors consider the energy-balance mechanisms in wireless networks. They suggest online distributed algorithms for energy-balance in different optimal network structures and numerically show their stability in particular setting. André Schumacher et al. ${ }^{[5]}$ propose an approximation algorithm for minimising the maximum network congestion as a modification to the DSR routing protocol.

Jaramillo et al. ${ }^{[6]}$ propose a reputation-based scheme called DARWIN to avoid retaliation situations after a node is falsely perceived as selfish to help restore cooperation quickly. Liu et al. ${ }^{[7]}$ suggest another reputation computation model to discover and prevent selfish behaviors by using familiarity values which allows nodes to obtain opinions with lower uncertainty values, helps nodes to recognize selfish nodes much earlier, and decreases the convergence time for isolating selfish nodes.

In [8] the authors investigate various ways of providing nodes near the edge of the network with preferential treatment in order to improve their credit balance and their throughputs. ICARUS proposed in [9] is a hybrid incentive mechanism, combining advantages of both reputation-based and credit-based mechanisms. The objective of ICARUS is to detect and punish selfish nodes efficiently and at the same time motivate nodes to cooperate by rewarding the packet forwarding.

\section{ENERGY BALANCING APPROACH BASED ON FORWARDING REFUSAL (EBFR)}

The basic working of EBFR which is an added module to any existing reactive routing protocols is as follows. Each node independently collects others' energy information then calculates the ratio of its own energy utilization to the whole network average. According to this ratio, each node periodically checks if it is overloaded or not, and selects one 
of the following three statuses: load balanced, overloaded and severe overloaded. In a different status, the node will take different data forwarding strategy to achieve energy balancing.

In order to adapt to node heterogeneity, residual energy level is computed according to the utilization of energy. Let $E_{i}$ denote the total energy of node $i$, and $R_{i}$ and $P_{i}$ denote the current residual energy and the number of buffered packets of node $i . F_{i}$ is the energy required to forward one packet in node $i$, and $U_{i}$ denotes the utilization of energy of node $i$. In EBFR, energy information refer to the utilization of energy which can be represented as shown below:

$$
U_{i}=\frac{R_{i}-F_{i} \times P_{i}}{E_{i}}
$$

The local view of energy information is a table in which each record corresponds to a node. A record contains the following four data items: <NodeID, Energy Utilization, Status, Timestamp>.

When a node receives an energy message, it will add or update record in local view of energy information. Energy Utilization in local view of energy information can be used to calculate the whole average of Energy Utilization which can be denoted by $U$. We define the ratio of its own energy utilization to the whole network average, which is denoted by $r_{i}$ as follow:

$$
r_{i}=\frac{U_{i}}{U}
$$

We define a lower threshold values $r_{l}$. If a node finds its $r_{i}$ lower than $r_{l}$, it considers itself load balanced. There is also an upper threshold values $r_{h}$ over which node considers itself severe overloaded. If $r_{l} \geqslant r_{i} \geqslant r_{h}$, the node $i$ is overloaded. An illustration of how we define statuses is shown in Figure 1 .

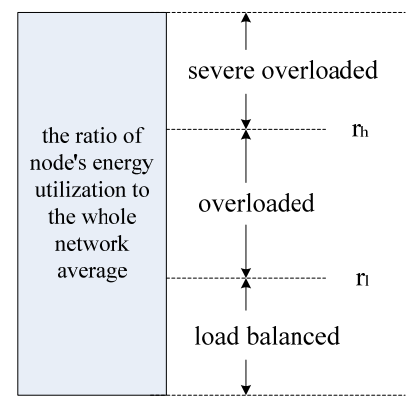

Figure1. Three statuses of node's energy utilization

If the node is load balanced, it broadcasts RREQs and forwards data packets the same as before. Else if the node is severe overloaded, it refuses to broadcast any RREQ and forward any data packet completely. Between the two statuses mentioned above, the node which is overloaded will forwards data packets the same as before, but ignore others' RREQs until is it load balanced.

\section{PROTOCOL-INDEPENDENT COOPERATIVE INCENTIVE SCHEME (PICIS)}

\section{A. Network model definition}

In this model, we assume all mobile nodes are rational and selfish, they hope to maximize its own profit and minimize the cost of its actions simultaneously. But we also believe that the interaction among nodes is reciprocal, they need others to forward its data packets. Therefore, the interaction between each pair of nodes can be defined as a two-player game.

We assume that there are $n$ nodes in MANET. The contribution of node $N_{i}$ can be denoted by a single number $c_{i}$ which is a continuous variable. For concreteness, we will take $c_{i}$ to be the number of data packets: data packets forwarded by $N_{i}$ over a fixed period of time, say a day. We encode the benefit of $N_{i}$ with a N-dimensional vector $B_{i}$, where $B_{i j}$ denotes how much the contribution made by $N_{j}$ to $N_{i}$. In general, $B_{i i}=0$ for all benefit vectors. Let $b_{i}$ denote the total benefit that $N_{i}$ can derive from the network, $b$ denote the average of $b_{i}$ for the whole network.

\section{B. Proposed scheme}

The requests of nodes which provides more services have a greater probability of being accepted. A node $N_{j}$ forwards data packets from source node $N_{i}$ with probability $p\left(c_{i}\right)$, and rejects it with probability $1-p\left(c_{i}\right) . p\left(c_{i}\right)$ is a monotonically increasing function of $c_{i}$, which has the following natural form:

$$
p(c)=\frac{e^{\varepsilon c}-1}{e^{\varepsilon c}+1}
$$

This function is very suitable for our needs, because $p(0)$ $=0$ and $\lim _{c \rightarrow+\infty} p(c)=1$. As shown in Figure 2, We can select the value of $\varepsilon$ according to the value range of $c$ : the value of $\varepsilon$ has negative correlation with the value of $c$.

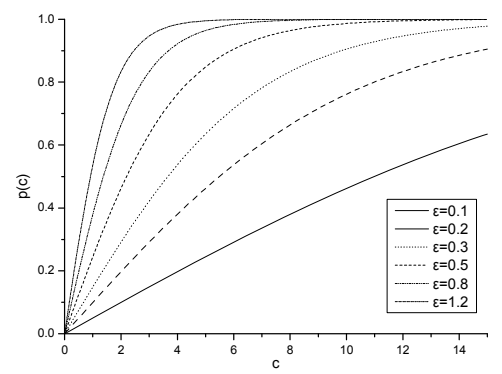

Figure 2. The function curve of $f(c)$

Definition 1. The total utility (TU) of network is the sum value of the contribution of each node

Definition 2. The individual utility (IU) of node $N_{i}$ is the total utility $I U_{i}$ that $N_{i}$ will derive by joining the network, that is:

$$
I U_{i}=b_{i}-c_{i}=\sum_{j=1}^{n} p\left(c_{i}\right) B_{i j} c_{j}-c_{i}=\sum_{j=1}^{n} \frac{e^{\varepsilon c_{i}}-1}{e^{\varepsilon c_{i}}+1} B_{i j} c_{j}-c_{i}
$$

When $N_{i}$ does not make any contribution to other nodes, that is, $c_{i}=0$, the probability of providing services to $N_{i}$ of 
other nodes will also be zero, that is, $p\left(c_{i}\right)=0$. On the contrary, when $c_{i}=\infty, p\left(c_{i}\right)=1$.

Therefore, we consider whether a Pareto optimal allocation of resources can be achieved from the rational allocation of resources in order to achieve the maximization of total utility of network. In the next Subsection, we will discuss the Nash equilibrium of the method proposed above combining with Bertrand's duopoly competition model ${ }^{[10]}$.

\section{Nash Equilibrium in the proposed scheme}

In incentives based on game theory, the strategy of nodes should achieve Nash equilibrium in order to achieve the purpose of inspiration for nodes. If the strategy can achieve Nash equilibrium, then the nodes can know the behavior selection strategy most favorable to their own to obtain greater benefits. As long as the utility functions are monotonic and concave, the most reasonable resource allocation scheme must exist. Not only each node is able to obtain maximum individual utility, the network can also maximize the total utility.

As mentioned above, the interaction between each pair of nodes can be defined as a two-player game. Assuming that there are only two nodes $N_{1}$ and $N_{2}$ in the network. We can find their individual utility function according to Equation 5:

$$
\left\{\begin{array}{l}
I U_{1}=p\left(c_{1}\right) B_{12} c_{2}-c_{1}=\frac{e^{\varepsilon c_{1}}-1}{e^{\varepsilon c_{1}}+1} B_{12} c_{2}-c_{1} \\
I U_{2}=p\left(c_{2}\right) B_{21} c_{1}-c_{2}=\frac{e^{\varepsilon c_{2}}-1}{e^{\varepsilon c_{2}}+1} B_{21} c_{1}-c_{2} .
\end{array}\right.
$$

According to Bertrand's duopoly competition model, we calculate partial derivative of individual utility function of node on its contribution as follow:

$$
\frac{\partial I U_{i}}{\partial c_{i}}=\sum_{j=1}^{n} \frac{2 e^{\varepsilon c_{i}}}{\left(e^{\varepsilon c_{i}}+1\right)^{2}} B_{i j} c_{j}-1
$$

Due to there are two nodes $N_{1}$ and $N_{2}$ in the network:

$$
\left\{\begin{array}{l}
\frac{\partial I U_{1}}{\partial c_{1}}=\frac{2 e^{\varepsilon c_{1}}}{\left(e^{\varepsilon c_{1}}+1\right)^{2}} B_{12} c_{2}-1 \\
\frac{\partial I U_{2}}{\partial c_{2}}=\frac{2 e^{\varepsilon c_{2}}}{\left(e^{\varepsilon c_{2}}+1\right)^{2}} B_{21} c_{1}-1 .
\end{array}\right.
$$

We obtain the solutions of Nash equilibrium equations:

$$
\left\{\begin{array}{l}
c_{1}=\frac{\left(e^{\varepsilon c_{2}}+1\right)^{2}}{2 e^{\varepsilon c_{2}} B_{21}} \\
c_{2}=\frac{\left(e^{\varepsilon c_{1}}+1\right)^{2}}{2 e^{\varepsilon c_{1}} B_{12}}
\end{array}\right.
$$

As shown in Figure 3 , the intersection $\left(c_{1}^{*}, c_{2}^{*}\right)$ of function curve of $c_{1}$ and $c_{2}$ is the optimal combination we seek. The benefit of two nodes will reach the Nash equilibrium here, then the total utility will obtain the maximum value.

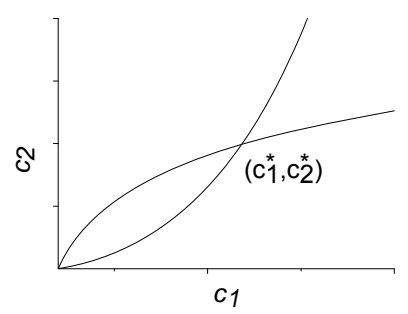

Figure 3. The Nash equilibrium contributions for the two nodes network

The above proof of Nash equilibrium of two nodes is also applicable to multiple nodes with the replacement of the parameters node number. In Ad hoc network with multinodes, there is also a Nash equilibrium to maximize the total utility of network. And the benefits of each node are always constantly adjusted according to its contribution to the system, to ensure that the network can always reach Nash equilibrium.

\section{SIMULATION AND ANALYSIS}

We present experimental results which evaluate our approach in a simulation environment built with NS-2 simulator. The performance study concerns three versions of DSR protocol: the classic DSR protocol, extended DSR with EBNG which is expressed as EBNG-DSR and extended DSR proposed in [5] which is named BMSR. The simulation parameters are given in Table 1.

TABLE 1. Parameters for performance simulation

\begin{tabular}{cc} 
Parameters & Value range \\
Number of mobile nodes & $128(27)$ \\
Range of simulation environment & $1000 \mathrm{~m} \times 1000 \mathrm{~m}$ \\
Transmission range of nodes & $300 \mathrm{~m}$ \\
Channel capacity & $3 \mathrm{Mbps}$ \\
Processing delay of data packets & $0.01 \mathrm{~s}$ \\
Initial energy & $360 \mathrm{~J}$ \\
Transmission energy consumption & $0.03 \mathrm{~J}$ \\
Receiving energy consumption & $0.02 \mathrm{~J}$ \\
\hline
\end{tabular}

Figure 4 demonstrates the Number of Alive Nodes under different packet rates. We see that BMSR and EBNG-DSR outperform DSR under different packet rates, because of the use of energy balancing approaches. The lifespan of network running EBNG-DSR is longer than those running classic DSR and BMSR, especially for high packet rates, that is, high network load. This shows that the energy balancing approaches improve network performance more significantly under high network load. 

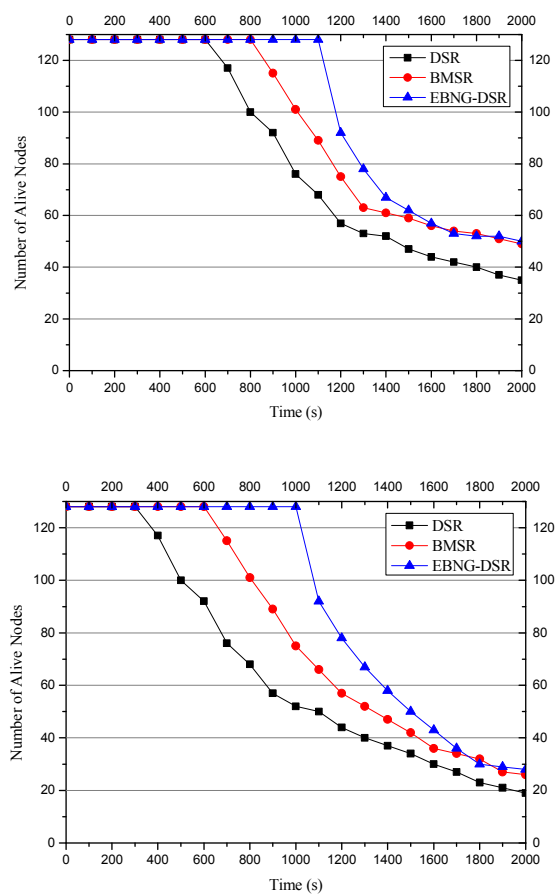

Figure 4. The Number of Alive Nodes under different packet rates (upper: 50 packets/min, lower: 100 packets/min).

In order to evaluate whether PICIS can effectively encourage selfish nodes, the nodes are divided into three categories: $25 \%$ of the nodes are selfish nodes, $25 \%$ are selfless nodes, and $50 \%$ of the nodes are mixed nodes.

Figure 5 shows the simulation results on the total utility of network against simulation time. The total utility of network which is extended with EBNG increases gradually over time until a stable value, while in the network without incentive mechanism, the total utility of network increases gradually over time in the first $500 \mathrm{sec}$, and then plummets until a stable value. On the contrary, in EBNG-DSR, nodes will gradually terminate the selfish behaviors and transform to selfless node in order to get better service.

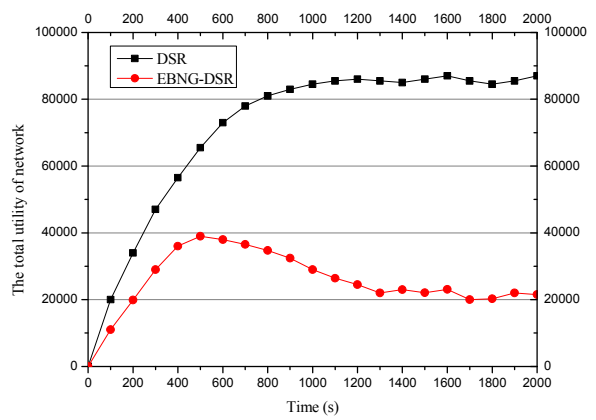

Figure 5 . Total utility of network against simulation time.

Figure 6 illustrates the simulation results on average endto-end delay against packet rate of node. Under low packet rate, the addition of EBNG increases average end-to-end delay as compared to the classic DSR, since when data packets are forwarded to overloaded and severe overloaded, the route discovery often need to be restarted and the packet delay is increased. However, the sacrifice in end-to-end delay can be ignored, due to considering EBNG's substantial enhancement in energy balancing. Meanwhile, under high packet rate, EBNG-DSR outperforms DSR and BMSR. Overall, we can conclude that EBNG improves the end-toend delay of DSR.

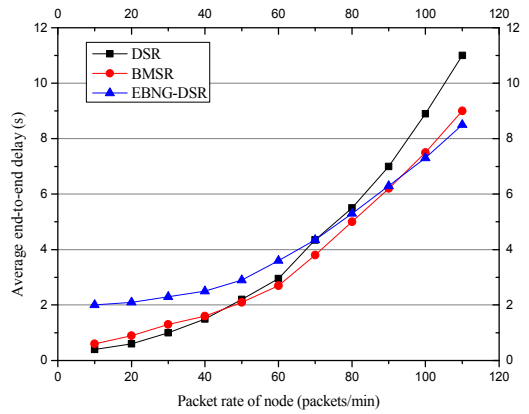

Figure 6. Average end-to-end delay against Packet rate of node.

The figure 7 proves that EBNG-DSR and BMSR provide better performance under high packet rate. A major factor that contributes to increased real-time packet delivery ratio is the decrease of the number of restarting route discovery due to the increase of the number of alive nodes. This shows that EBNG performs well under increasing network load.

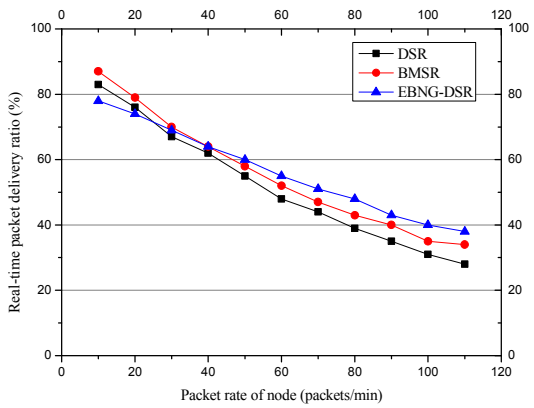

Figure 7. Real-time packet delivery ratio against Packet rate of node.

\section{CONCLUSIONS}

We have presented an energy balancing approach called EBNG that performs load and energy balancing. EBNG combines an Energy Balancing Approach Based on Forwarding Refusal named EBFR with a ProtocolIndependent Cooperative Incentive Scheme named PICIS. In EBFR, each node independently collects energy information and periodically checks if it is overloaded or not, then takes different data forwarding strategy to achieve energy balancing. Meanwhile, due to the inherent selfishness and greed of mobile nodes, PICIS is used to inhibit the proliferation of free riding behavior. The existence of Nash equilibrium shows that not only each node is able to obtain maximum individual utility, the network can also maximize 
the total utility. Simulation results showed that EBNG can maintain better performance without sacrificing the overall packet delivery performance under increasing network load although initially it is inferior due to its additional complexity. As future work we consider the further experimentation in order to optimize the energy balancing approach for a wide variety of network topologies and scenarios.

\section{ACKNOWLEDGMENT}

This research was supported by the National Natural Science Foundation of China (Grant No. 61272454).

\section{REFERENCES}

[1] D.P. Agrawal, Q.A. Zeng, "Introduction to wireless and mobile systems", Ad Hoc and Sensor Networks, Brooks/Cole-Thomson Learning, pp.297-348, 2003.

[2] W.J. Adams, G.C. Hadjichristofi, N.J. Davis, "Calculating a node's reputation in a mobile ad hoc network", 24th IEEE International Performance Computing and Communications Conference, pp.303$307,2005$.

[3] Y. Yoo, S. Ahn, D.P. Agrawal, "Impact of a simple load balancing approach and an incentive-based scheme on MANET performance", Journal of Parallel and Distributed Computing, vol.70, no.2, pp.71-83, 2010 .
[4] Aubin Jarrya, Pierre Leonea, Sotiris Nikoletseasb, Jose Rolim, "Optimal data gathering paths and energy-balance mechanisms in wireless networks", Ad Hoc Networks, vol.9, no.6, pp.1036-1048, 2011.

[5] André Schumacher, Harri Haanpää, Satu Elisa Schaeffer, Pekka Orponen, "Load balancing by distributed optimisation in ad hoc networks", Proceedings of The 2nd International Conference on Mobile Ad-hoc and Sensor Networks, vol.4325, pp.873-884, 2006.

[6] Juan José Jaramillo, R. Srikant, "A game theory based reputation mechanism to incentivize cooperation in wireless ad hoc networks", Ad Hoc Networks, vol.8, no.4, pp.416-429, 2010.

[7] Yining Liu, Keqiu Li, Yingwei Jin, Yong Zhang, Wenyu Qu, "A novel reputation computation model based on subjective logic for mobile ad hoc networks", Future Generation Computer Systems, vol.27, no.5, pp.547-554, 2011.

[8] Jean-Marc Robert, Hadi Otrok, Abdelkarim Chriqi, "RBC-OLSR: Reputation-based clustering OLSR protocol for wireless ad hoc networks", Computer Communications, vol.35, no.4, pp.487-499, 2012.

[9] Dimitris E. Charilas, Konstantinos D. Georgilakis, Athanasios D. Panagopoulos, "ICARUS: hybrId inCentive mechAnism for coopeRation stimUlation in ad hoc networkS", Ad Hoc Networks, In Press, 2012.

[10] Terrence M. Belton, "A model of duopoly and meeting or beating competition", International Journal of Industrial Organization, vol.5, no.4, pp.399-417, 2005. 